\title{
Influência da aplicação de um programa de estiramentos estáticos, após contracções excêntricas, nas manifestações clínicas e bioquímicas de lesão muscular esquelética
}

\section{R. Torres ${ }^{1}$ \\ P. Carvalho ${ }^{2}$}

J.A. Duarte ${ }^{3}$

https://doi.org/10.5628/rpcd.05.03.274

\author{
${ }^{1}$ Instituto Politécnico da Saúde Norte \\ Escola Superior de Saúde de Vale do Sousa \\ Departamento de Fisioterapia \\ ${ }^{2}$ Centro de Medicina Desportiva do Norte \\ ${ }^{3}$ Universidade do Porto \\ Faculdade de Desporto, CIAFEL \\ Portugal
}

\section{ABSTRACT}

Effects of a static stretching program on clinical and biochemical markers of muscle damage induced by eccentric exercise.

Este estudo teve como objectivos determinar se o estiramento estático do músculo quadriceps, (i) por si só, induz agressão no músculo íntegro, e se (ii) efectuado após o exercício físico agrava as manifestações clínicas e bioquímicas da agressão muscular. Vinte e sete sujeitos jovens do sexo masculino, sedentários, foram divididos por 3 grupos e submetidos: Grupo 1 - a um programa de estiramentos estáticos; Grupo 2 - a um programa de contracções excêntricas; e Grupo 3 - a um programa de contracções excêntricas (idêntico ao Grupo 2) seguido por um programa de estiramentos (idêntico ao Grupo 1). Foram avaliados parâmetros clínicos e bioquímicos imediatamente antes do exercício e 1, 24, 48, 72, 96 horas após. A avaliação clínica compreendeu a quantificação da dor à palpação, da circunferência da coxa, do Peak Torque Excêntrico Máximo. A avaliação bioquímica compreendeu a quantificação da actividade plasmática da Creatina Kinase e da Transaminase Glutâmica Oxaloacética. Não foram obser vadas alterações clínicas ou bioquímicas, ao longo do tempo, no grupo submetido unicamente ao programa de estiramento; obser vou-se, ainda, que as manifestações de lesão muscular foram idênticas para o grupo 2 e grupo 3 . Em conclusão, o estiramento estático, por si só, não parece induzir agressão muscular esquelética no quadriceps íntegro e, quando realizado neste músculo após a agressão pelo exercício excêntrico, não parece agravar as manifestações clínicas e bioquímicas da miopatia do exercício.

Palavras-chave: exercício excêntrico, estiramento estático, agressão muscular, miopatia do exercício, desconforto muscular.
The aims of this study were to determine if the quadriceps static stretching, (i) alone, induces aggression in the integral muscle, and if (ii) done after physical exercise, intensifies the muscular aggression clinical and biochemical manifestations. Twenty-seven young sedentary male subjects were divided in three groups. A protocol of static stretching was applied to group 1; a protocol of eccentric contractions was applied to group 2, while group 3 followed a protocol consisting of eccentric contractions (similar to the one performed by group 2) and stretching (identical to group 1). Clinical and biochemical parameters were evaluated immediately before and 1, 24, 48, 72 and 96 hours after exercise. The clinical evaluation included the quantification of pain induced by palpation, thigh girth, and Eccentric Maximal Peak Torque determination. The biochemical evaluation consisted on the quantification of plasmatic kinase creatine and oxaloacetic glutamic transaminase activities. No clinical or biochemical alterations were observed during the time in the group that only followed the stretching protocol. The muscular damage was identical in group 2 and group 3. Concluding, static stretching by itself does not seem to induce a skeletal muscular aggression in an integral quadricep s. When applied in this muscle after the aggression caused by eccentric exercise, it does not seem to intensify exercise miopathy clinical and biochemical manifestations.

Key Words: eccentric exercise, static stretching, muscular aggression, exercise miopathy, muscle soreness. 


\section{INTRODUÇÃO}

Está hoje bem descrito que o exercício físico, particularmente se inabitual e/ou envolvendo contracções excêntricas, constitui uma agressão para os músculos esqueléticos solicitados $(2,3,10,11,27,34,37)$. Esta agressão induz um quadro anatomo -patológico caracterizado por uma natureza necrótica, catabólica e inflamatória, denominado, por alguns autores, por miopatia do exercício (MEx) (48).

De facto, após a realização de exercício intenso é possível obser var-se nos músculos solicitados alterações estruturais e ultra-estruturais (20), tais como irregularidades no padrão estriado das fibras musculares, o aparecimento de núcleos centrais, a disrupção e vacuolização sarcoplasmática, o aumento do volume mitocondrial, o aparecimento de áreas de necrose segmentar, o edema celular e a disrupção das linhas $\mathrm{Z}$ com extensão do seu material para as bandas I adjacentes $(4,10,15,20)$.

Lieber et al. (26) defendem, também, que o exercício excêntrico poderá levar à disrupção de alguns filamentos intermediários, como a titina, responsáveis pela manutenção da sobreposição da actina e miosina. A titina, parece desempenhar uma importante tarefa na transmissão da tensão mecânica às linhas $\mathrm{Z}$ aquando do estiramento passivo do músculo esquelético (50). Assim, por este mecanismo, o fenómeno de sobrecarga mecânica, verificado ao nível das linhas Z, poderá ser idêntico durante o estiramento e a contracção excêntrica (28).

A realização de programas de estiramento, antes e/ou após a realização de exercício intenso, é extremamente habitual na prática desportiva $(1,21)$. A sua aplicação tem como finalidade prevenir ou diminuir os efeitos agressivos do exercício (1, 13, 28, 40), prevenir lesões traumáticas desportivas (1, 8, 25), aumentar a recuperação muscular (45), aumentar a amplitude articular $(1,21,31)$ e melhorar a performance $(8,21)$.

No entanto, a literatura, apesar de vasta nesta temática, está longe de apresentar consenso nos efeitos do estiramento ao nível do músculo íntegro $(28,47)$, ou quando agredido pelo exercício (21).

Entre os autores que defendem um efeito benéfico do estiramento perante a agressão muscular encontra-se deVries (13). Este autor defende que o estiramento realizado após o exercício contribui para a redução do espasmo muscular instalado, aliviando, assim, a manifestação clínica imposta pela agressão do exercício. Já para Bobbert et al. (5), o benefício do estiramento poderá consistir na capacidade de dispersar o edema verificado após o exercício, contribuindo para a recuperação dos tecidos e para a atenuação do quadro clínico. O estiramento poderá ainda, através do estímulo mecânico imposto pelo estiramento, aumentar o limiar de excitabilidade das terminações nervosas das fibras do tipo III e IV, contribuindo para uma redução dos sintomas da agressão muscular (2).

No entanto, os trabalhos de Buroker e Schwane (7), High et al. (22), Lund et al. (28), Rodenburg et al. (40) e Wessel e Wan (52) não confirmam tais benefícios.

O estiramento aplicado imediatamente após a realização de um exercício agressivo poderá mesmo, segundo Lund et al. (28), ter um papel negativo no quadro clínico e bioquímico característico da MEx. O trabalho realizado por Smith et al. (47), com o objectivo de determinar qual o efeito da aplicação do estiramento estático e balístico inabitual no músculo íntegro, verificou uma manifestação do quadro clínico e bioquímico idêntica à da agressão muscular induzida pelo exercício excêntrico. Estes resultados, ajudam a suportar a hipótese do estiramento, quando aplicado após a realização de exercício físico intenso ou inabitual, agravar o quadro clínico e bioquímico da MEx.

Porque não existe actualmente consenso quanto ao papel que os programas de estiramento muscular poderão ter, quer no músculo íntegro, quer naquele submetido a exercício agressivo, este trabalho teve como objectivos determinar se o estiramento estático do músculo quadriceps, (1) por si só, induz agressão no músculo íntegro, e se (2) efectuado após o exercício físico, agrava as manifestações clínicas e bioquímicas da agressão muscular.

Assim, partiu-se para este trabalho acreditando nas hipóteses suportadas por Lund et al. (28) e Smith et al. (47), em que (1) o estiramento estático inabitual induz, por si só, agressão muscular e que, (2) quando realizado imediatamente após a finalização do exercício excêntrico extenuante, agrava o quadro clínico e bioquímico da MEx. 


\section{MATERIAL E MÉTODOS}

Após obtenção do consentimento informado, considerando a "Declaração de Helsínquia" da

Associação Médica Mundial, constituiu-se uma amostra com 30 sujeitos, jovens do sexo masculino, maturos sexualmente.

Para a inclusão dos sujeitos no estudo, definiram-se como critérios: (i) serem do sexo masculino, com idades entre os 18 e os 40 anos; (ii) serem sedentários, sem actividade física regular; (iii) não terem frequentado, nos últimos 3 meses, quaisquer programas de estiramento muscular ; (iv) não terem qualquer comprometimento músculo -esquelético, ao nível do membro inferior, que impossibilitasse a realização dos exercícios do protocolo experimental; e $(v)$ não terem sofrido qualquer sensação retardada de desconforto muscular nos últimos 3 meses, no músculo quadriceps do membro dominante.

Os sujeitos seriam excluídos do estudo, caso: (i) apresentassem em repouso valores bioquímicos sanguíneos [Creatina Kinase $(\mathrm{CK}) \geq 60 \mathrm{U} / \mathrm{L}$,

Transaminase Glutâmica Oxaloacética (TGO) $\geq 20$ U/L] sugestivos de uma agressão muscular induzida por agressão recente, ou (ii) realizassem qualquer inter venção à margem do protocolo experimental, como, aplicação de calor, frio, massagem, ingestão de analgésicos, execução de estiramento ou de exercício físico inabitual.

Os 30 elementos, que constituíam inicialmente a amostra, foram distribuídos aleatoriamente por 3 grupos: Grupo $1(\mathrm{G} 1, \mathrm{n}=10)$, Grupo $2(\mathrm{G} 2, \mathrm{n}=10)$ e Grupo 3 (G3, n=10). Retiram-se, no entanto, 2 elementos ao grupo 2 e 1 elemento ao grupo 3, por cumprirem alguns dos critérios de exclusão. As características gerais de cada grupo estão ilustradas no quadro 1.

Quadro 1 - Valores médios, com respectivos desvios-padrão, da idade (anos], do peso $(\mathrm{Kg}$ ), da altura $(\mathrm{cm}$ ), do índice de massa corporal (IMC, $\mathrm{Kg} / \mathrm{m} 2$ ] e do peak torque excêntrico máximo (PTEM, N.m] nos três grupos estudados, avaliados imediatamente antes do protocolo experimental.

\begin{tabular}{|l|c|c|c|c|c|}
\hline & Grupo 1 & Grupo 2 & Grupo 3 & F & P \\
\hline $\begin{array}{l}\text { N }^{0} \\
\text { sujeitos }\end{array}$ & 10 & 8 & 9 & & \\
\hline Idade & $20,4 \pm 2,41$ & $23,6 \pm 4,34$ & $21,2 \pm 2,22$ & 2,531 & 0,101 \\
\hline Peso & $70,4 \pm 6,09$ & $77,6 \pm 11,30$ & $70,8 \pm 6,03$ & 1,001 & 0,382 \\
\hline Altura & $177,2 \pm 6,35$ & $175,6 \pm 6,39$ & $173,5 \pm 3,64$ & 2,184 & 0,184 \\
\hline IMC & $22,4 \pm 1,69$ & $24,7 \pm 1,20$ & $23,7 \pm 1,29$ & 2,120 & 0,142 \\
\hline PTEM & $284,3 \pm 66,89$ & $352,6 \pm 76,49$ & $303,1 \pm 59,96$ & 1,333 & 0,283 \\
\hline
\end{tabular}

\section{PROCEDIMENTOS}

Os 3 grupos inter venientes no estudo foram submetidos a um programa de exercícios de estiramento e/ou a um programa de contracções excêntricas, aplicado ao músculo quadriceps do membro dominante. Assim:

G1 - foi submetido a um programa de estiramentos; G2 - foi submetido a um programa de contracções excêntricas; e

G3 - foi submetido a um programa de contracções excêntricas (idêntico a G2), seguido por um programa de estiramentos (idêntico a G1).

Do protocolo experimental aplicado a cada grupo fizeram parte vários momentos de avaliação ao longo do tempo. A figura 1 apresenta a sequência cronológica dos 6 momentos de avaliação previstos no protocolo experimental.

Em cada momento de avaliação, foram recolhidas medidas clínicas e sangue capilar, para análise do efeito dos programas de exercício excêntrico e de estiramento, ao nível das variáveis dependentes.

\section{Programa de exercícios de estiramento}

O programa de exercícios de estiramento, realizado no músculo quadriceps do membro dominante, compreendeu 10 estiramentos passivos, com duração de 30 segundos cada. O tempo de repouso entre estiramentos foi de 10 segundos. A duração total deste programa foi de 6,5 minutos.

A execução do estiramento passivo foi sempre realizada pelo mesmo investigador que, de acordo com o sugerido na literatura $(23,28,40)$, aplicou suavemente o estiramento até que fosse sentida resistência ao alongamento e/ou que o sujeito manifestasse qualquer desconforto.

Para a realização do estiramento, adaptou-se o procedimento descrito por Anderson e Burke (1): O sujeito encontrava-se de pé, frente a um espaldar, com o joelho do membro não dominante flectido cerca de $45^{\circ}$ e com o peso do corpo suportado na berma de uma marquesa, conforme a figura 2 . 


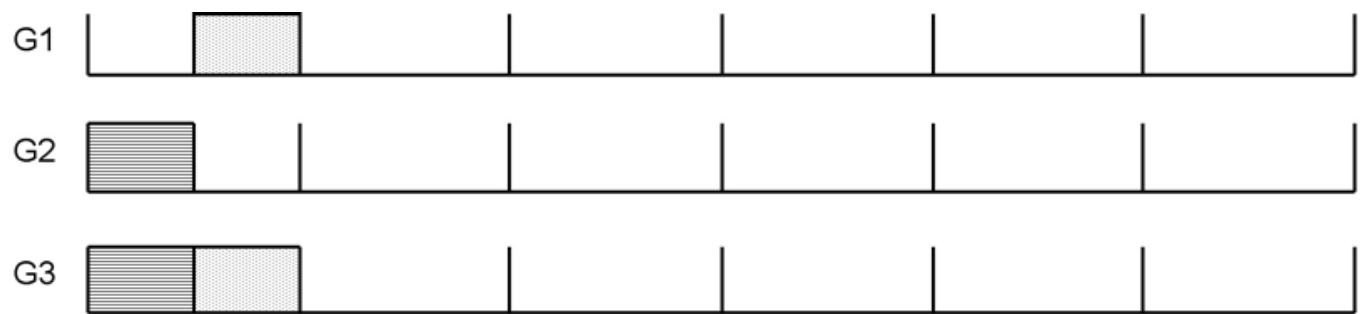

\begin{tabular}{|c|c|c|c|c|c|c|}
\hline $\begin{array}{l}\text { Antes } \\
\text { - Momento } \\
\text { e avaliacão }\end{array}$ & 0 Horas & $\begin{array}{c}1 \text { Hora } \\
2^{\circ} \text { Momento } \\
\text { de avaliação }\end{array}$ & $\begin{array}{c}24 \text { Horas } \\
3^{\circ} \text { Momento } \\
\text { de avaliação }\end{array}$ & $\begin{array}{c}48 \text { Horas } \\
4^{\circ} \text { Momento } \\
\text { de avaliação }\end{array}$ & $\begin{array}{c}72 \text { Horas } \\
5^{\circ} \text { Momento } \\
\text { de avaliação }\end{array}$ & $\begin{array}{c}96 \text { Horas } \\
6^{\circ} \text { Momento } \\
\text { de avaliaçãa }\end{array}$ \\
\hline
\end{tabular}

Programa de exercícios de estiramento

Programa de exercícios excêntricos

Figura 1 - Apresentação da sequência cronológica do protocolo experimental aplicado aos diferentes grupos (G1 - Grupo1; G2 - grupo 2; G3 - Grupo 3).

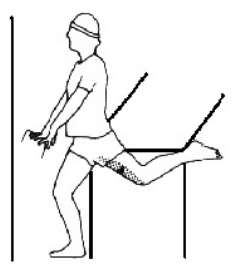

Figura 2. Posicionamento para realização do programa de exercícios de estiramento (adaptado de Anderson e Burke, 1991).

O membro dominante encontrava-se totalmente relaxado, o investigador começava por flectir o joelho do sujeito até ao máximo de flexão; caso esta amplitude articular não provocasse um estiramento ao músculo quadriceps que manifestasse resistência e/ou desconforto muscular, a extensão da articulação coxo-femural seria a segunda componente implementada para aumentar o estiramento.

\section{Programa de exercícios excêntricos}

Seleccionou-se um programa de exercícios com contracções excêntricas, para induzir agressão ao músculo quadriceps do membro dominante dos sujeitos $(18,23,29)$. Este programa foi realizado num dinamómetro isocinético Biodex, System $3^{\circledR}$, e foi precedido por um período de activação geral em cicloergó- metro (Monark ${ }^{\text {mu }}$ E-824), com uma resistência correspondente a $2 \%$ do peso corporal, durante um período de 5 minutos (23). O programa de exercícios excêntricos compreendeu 2 séries de contracções excêntricas até à fadiga, separadas por 30 segundos, a $80 \%$ do peak torque máximo. Em cada série a velocidade de contracção foi de $60^{\circ}$ por segundo e o tempo de repouso entre cada contracção de 1 segundo. A amplitude de movimento estava compreendida entre $20^{\circ}$ e $90^{\circ}$ de flexão do joelho. Aquando da realização das contracções excêntricas, os sujeitos foram sentados na cadeira do dinamómetro isocinético, procedendo -se à sua estabilização com cintos ao nível da coxa, abdómen e tronco, para prevenir eventuais compensações. Os membros superiores encontravam-se cruzados ao nível do tronco (38). Após o posicionamento, os sujeitos realizaram alguns movimentos, para habituação e aprendizagem do exercício, com resistência moderada.

\section{Avaliação clínica}

A avaliação clínica foi efectuada em todos os momentos de avaliação (figura 1) e teve por objectivos quantificar a dor à palpação, a circunferência da coxa e a função muscular. 
Os sujeitos encontravam-se em decúbito dorsal, com os joelhos em extensão e com musculatura da coxa relaxada.

A dor induzida por palpação referida pelos sujeitos, realizada no músculo quadriceps submetido ao exercício, foi medida através da Escala Visual Analógica (EVA) $(18,37,41)$. A palpação foi sempre realizada pelo mesmo investigador com a preocupação de realizar, em todos os momentos de avaliação e para todos os sujeitos, níveis de pressão idêntica. Foram também realizadas medições da circunferência da coxa dos sujeitos em 2 zonas diferentes. Para tal, marcou-se com caneta resistente à água o pólo superior da rótula e a espinha ilíaca antero -superior (EIAS), medindo -se esta distância e dividindo -a em 6 partes. As medições foram realizadas com fita métrica e as zonas de medição foram marcadas com uma caneta resistente à água, para facilitar as medições seguintes. Sempre que necessário, nas várias medições, foram reforçadas as marcas.

A circunferência distal e circunferência proximal foram definidas, respectivamente, a $1 / 6$ e $2 / 6$ da distância entre o pólo superior da rótula e a EIAS. As medições foram sempre realizadas pelo mesmo investigador, com o objectivo de aumentar a consistência dos resultados. No sentido de determinar a fiabilidade das medições da circunferência da coxa, foi realizado antes do início do protocolo experimental, um teste-reteste com 48 horas de intervalo, com 10 sujeitos da amostra. Foram calculados os Coeficientes de Correlação Intra-classe (CCI) para ambos os níveis de medição. Os resultados obtidos apresentaram-se elevados (CCI $=0.99$ circunferência proximal e $\mathrm{CCI}=0,98$ circunferência distal), demonstrando uma boa consistência deste procedimento de medição. Para a caracterização da função muscular utilizou-se o Peak Torque Excêntrico Máximo (28).

Este foi avaliado através de 3 contracções excêntricas máximas no músculo quadriceps do membro dominante, separadas por 1 minuto. O valor do peak torque excêntrico mais elevado, em N.m, foi utilizado como indicador da função muscular. Estas medições foram realizadas a uma velocidade angular de $60 \%$ Seg., no Biodex, system $3^{\circledR}$. A amplitude de movimento escolhida foi de $20^{\circ}$ a $90^{\circ}$ do joelho.

Os procedimentos de preparação para a medição das medições do peak torque excêntrico, anteriormente descritos aquando da descrição do programa de exercício excêntrico, foram igualmente aplicados a todos os sujeitos, nos vários momentos de avaliação.

\section{Avaliação bioquímica}

A avaliação bioquímica foi efectuada em todos os momentos de avaliação (figura 1) e teve por objectivo averiguar a actividade plasmática das enzimas musculares CK e TGO.

Para determinação da actividade enzimática da CK no plasma foram retirados 32 microlitros de sangue capilar do lóbulo auricular dos sujeitos, após ter sido realizada limpeza ao lóbulo da orelha de cada sujeito com álcool etílico a 95\%.

De seguida, após secagem com algodão, o lóbulo foi picado com uma lanceta esterilizada e o sangue foi drenado para um tubo capilar heparinizado (Cat $\mathrm{n}^{\circ}$ 955053202 Reflotron ${ }^{\circledast}$ ).

O sangue foi imediatamente pipetado para uma tira reactiva de CK (Cat n ${ }^{\circ} 1126695$ Reflotron $^{\star}$ ) e colocada no Reflotron Analyser ${ }^{\oplus}$, da Boehringer

Mannheim, para a análise da actividade desta enzima muscular. Este procedimento foi repetido, de seguida, para análise da actividade enzimática da TGO (Cat $\mathrm{n}^{\circ} 745120$ Reflotron $^{\oplus}$ ).

\section{Tratamento estatístico}

No tratamento dos dados, foram utilizadas as medidas de dispersão e de tendência central no âmbito da estatística descritiva, nomeadamente a média e o desvio -padrão.

Os resultados das variáveis dor à palpação, CK e TGO, foram tratados em valores absolutos.

As restantes variáveis foram tratadas em valores absolutos (para comparações intra-grupais) e relativos (para comparações inter-grupais), através da fórmula:

\section{(Valor final - Valor inicial) X100 Valor inicial}

Após análise exploratória dos dados e estudo da normalidade de distribuição das variáveis, foi efectuada a análise inferencial dos resultados através de testes paramétricos para todas as variáveis de distribuição normal (Anova multifactorial, para comparação inter grupal para cada momento e Anova de medidas repetidas, para comparações intra-grupais ao longo do 
tempo) e de testes não-paramétricos (Krusk al-

Wallis) para aquelas de distribuição anormal (actividades da CK e da TGO). Quando na análise inferencial paramétrica eram evidenciadas diferenças, recorreu-se ao teste de múltiplas comparações de Scheffé. Estabeleceu-se um nível de significância de $\alpha=0,05$.

\section{APRESENTAÇÃO DOS RESULTADOS \\ Avaliação clínica}

Os valores médios absolutos e a análise estatística intragrupal do comportamento da dor à palpação, circunferência distal e proximal da coxa, força, CK e TGO, ao longo dos vários momentos de avaliação do protocolo experimental, encontram-se ilustrados no quadro 2 .

Quadro 2 - Médias e respectivos desvios-padrão dos valores absolutos da dor à palpação, circunferência distal e proximal da coxa (cm), força (N.m), creatina kinase (CK, U/I), transaminase glutâmica oxaloacética (TGO, U/I), nos vários momentos do protocolo experimental, nos 3 grupos estudados.

\begin{tabular}{|c|c|c|c|c|c|c|c|}
\hline Medidas & Grupo & Antes & 1 Hora & 24 Horas & 48 Horas & 72 Horas & 96 Horas \\
\hline $\begin{array}{l}\text { Dor à } \\
\text { palpação }\end{array}$ & $\begin{array}{l}G 1 \\
G 2 \\
G 3\end{array}$ & $\begin{array}{l}0.0 \pm 0.0 \\
0.0 \pm 0.0 \\
0.0 \pm 0.0\end{array}$ & $\begin{array}{l}0.0 \pm 0.0 \\
0.6 \pm 0.5 a \\
0.6 \pm 0.7 \text { bce }\end{array}$ & $\begin{array}{l}0.0 \pm 0.0 \\
3.2 \pm 0.6 \mathrm{a} \\
4.1 \pm 1.2 \mathrm{a}\end{array}$ & $\begin{array}{l}0.0 \pm 0.0 \\
5.3 \pm 1.5 \text { ade } \\
5.7 \pm 1.1 .8 \text { ae }\end{array}$ & $\begin{array}{l}0.0 \pm 0.0 \\
2.8 \pm 1.6 \mathrm{a} \\
3.6 \pm 2.0 \mathrm{a}\end{array}$ & $\begin{array}{l}0.0 \pm 0.0 \\
1.3 \pm 1.2 \\
2.0 \pm 1.8\end{array}$ \\
\hline $\begin{array}{l}\text { Circunferência } \\
\text { distal }\end{array}$ & $\begin{array}{l}G 1 \\
G 2 \\
G 3\end{array}$ & $\begin{array}{l}41.8 \pm 2.8 \\
44.6 \pm 4.0 \\
43.4 \pm 3.6\end{array}$ & $\begin{array}{l}41.8 \pm 2.8 \\
44.9 \pm 4.0 \mathrm{c} \\
43.6 \pm 3.6 \mathrm{bcd}\end{array}$ & $\begin{array}{l}41.9 \pm 2.9 \\
45.3 \pm 4.0 \mathrm{a} \\
44.7 \pm 3.6 \mathrm{ae}\end{array}$ & $\begin{array}{l}41.9 \pm 2.9 \\
45.4 \pm 4.0 \mathrm{a} \\
44.2 \pm 3.7 \text { ae }\end{array}$ & $\begin{array}{l}41.8 \pm 2.8 \\
45.2 \pm 4.0 \mathrm{a} \\
44.0 \pm 3.6 \mathrm{a}\end{array}$ & $\begin{array}{l}41.8 \pm 2.8 \\
45.0 \pm 4.0 \mathrm{a} \\
43.7 \pm 3.6\end{array}$ \\
\hline $\begin{array}{l}\text { Circunferência } \\
\text { proximal }\end{array}$ & $\begin{array}{l}G 1 \\
G 2 \\
G 3\end{array}$ & $\begin{array}{l}48.7 \pm 3.2 \\
51.8 \pm 4.3 \\
49.9 \pm 4.8\end{array}$ & $\mid \begin{array}{l}48.8 \pm 3.3 \\
52.0 \pm 4.5 \text { bcde } \\
50.0 \pm 4.7 \mathrm{~cd}\end{array}$ & $\begin{array}{l}48.6 \pm 3.2 \\
52.6 \pm 4.6 \mathrm{a} \\
50.6 \pm 4.8 \mathrm{a}\end{array}$ & $\begin{array}{l}48.7 \pm 3.2 \\
53.0 \pm 4.6 \mathrm{a} \\
50.8 \pm 4.7 \mathrm{a}\end{array}$ & $\begin{array}{l}48.7 \pm 3.2 \\
52.9 \pm 4.7 \mathrm{a} \\
50.7 \pm 4.7 \mathrm{a}\end{array}$ & $\mid \begin{array}{l}48.7 \pm 3.2 \\
52.7 \pm 4.4 a \\
50.3 \pm 4.9\end{array}$ \\
\hline Força & $\begin{array}{l}G 1 \\
G 2 \\
G 3\end{array}$ & $\begin{array}{l}284.2 \pm 66.9 \\
352.6 \pm 76.5 \\
303.1 \pm 60.0\end{array}$ & $\begin{array}{l}282.0 \pm 66.2 \\
276.1 \pm 66.3 \text { acde } \\
231.1 \pm 49.1 \text { abcde }\end{array}$ & $\begin{array}{l}284.7 \pm 64.6 \\
294.9 \pm 65.7 \text { ae } \\
266.5 \pm 57.5 \text { ade }\end{array}$ & $\begin{array}{l}285.7 \pm 67.7 \\
308.2 \pm 65.5 \text { ae } \\
275.3 \pm 54.2 \text { ae }\end{array}$ & $\begin{array}{l}284.0 \pm 70.0 \\
318.1 \pm 75.1 \mathrm{a} \\
285.7 \pm 63.7\end{array}$ & $\begin{array}{l}285.0 \pm 67.8 \\
340.0 \pm 70.7 \\
302.6 \pm 59.9\end{array}$ \\
\hline CK & $\begin{array}{l}G 1 \\
G 2 \\
G 3\end{array}$ & $\begin{array}{l}37.5 \pm 12.4 \\
34.8 \pm 4.6 \\
41.9 \pm 8.8\end{array}$ & $\begin{array}{l}38.8 \pm 9.3 \\
41.0 \pm 7.7 \\
40.7 \pm 9.2\end{array}$ & $\begin{array}{l}52.3 \pm 28.4 \\
91.6 \pm 54.5 \\
136.3 \pm 94.7\end{array}$ & $\begin{array}{l}40.8 \pm 12.6 \\
275.4 \pm 380.9 \\
374.5 \pm 380.7\end{array}$ & $\begin{array}{l}40.5 \pm 15.3 \\
253.2 \pm 362.7 \\
312.1 \pm 273.9\end{array}$ & $\begin{array}{l}37.0 \pm 9.8 \\
417.6 \pm 668.2 \\
341.7 \pm 344.6\end{array}$ \\
\hline TGO & $\begin{array}{l}G 1 \\
G 2 \\
G 3\end{array}$ & $\begin{array}{l}10.3 \pm 1.7 \\
10.5 \pm 2.0 \\
12.0 \pm 3.7\end{array}$ & $\begin{array}{l}9.7 \pm 1.2 \\
12.4 \pm 2.9 \\
9.3 \pm 1.8\end{array}$ & $\begin{array}{l}11.8 \pm 1.7 \\
11.2 \pm 1.7 \\
11.6 \pm 2.4\end{array}$ & $\begin{array}{l}10.6 \pm 1.5 \\
22.1 \pm 16.90 \\
17.1 \pm 18.0\end{array}$ & $\begin{array}{l}9.2 \pm 1.6 \\
29.7 \pm 30.0 \\
32.9 \pm 35.2\end{array}$ & $\begin{array}{l}11.4 \pm 1.1 \\
33.6 \pm 36.3 \\
38.9 \pm 51.9\end{array}$ \\
\hline
\end{tabular}

Como se pode verificar, através do quadro 2, o grupo G1 apresentou ausência de dor à palpação, durante as várias medições efectuadas durante o estudo. Quanto aos grupos G2 e G3, estes apresentaram comportamentos bastante semelhantes. Ambos apresentaram inícios de dor na $1^{\text {a }}$ hora, manifestaram "picos" máximos de dor às 48 horas e mantiveram ainda dor à palpação às 96 horas.
Comparativamente à situação pré-exercício (Antes), o grupo G2 apresentou entre a 1 e as 72 horas valores de dor à palpação estatisticamente significativos $(\mathrm{p}<0.05)$. O grupo G3 apresentou valores significativos apenas entre as 24 e 72 horas $(p<0.05)$.

A figura 3 e representa a análise estatística intergrupal do comportamento da dor à palpação, nos vários momentos de avaliação efectuados durante o estudo. 
Escala Visual Analógica (0-10)

...... Grupo 1

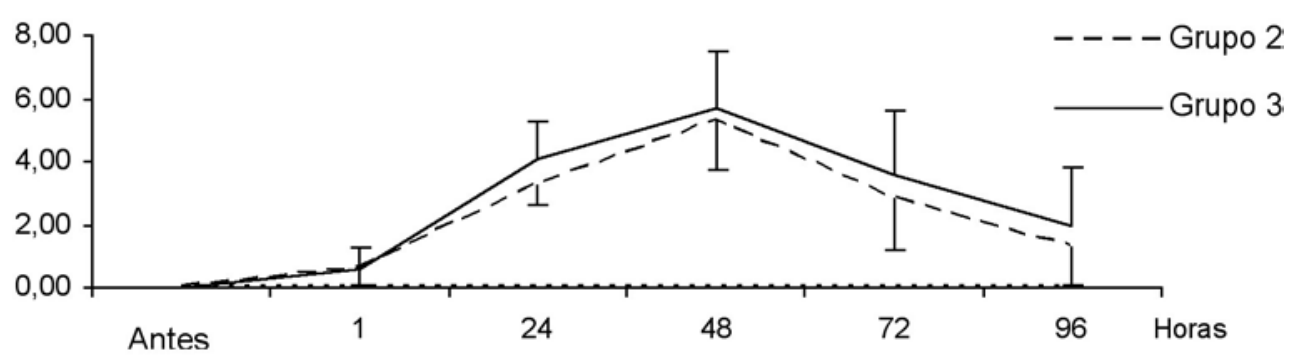

\begin{tabular}{l|l|l|l|l|l|l} 
p< $0,05:$ & G1 vs G2 & G1 vs G2 & G1 vs G2 & G1 vs G2 & G1 vs G3 \\
\hline & G1 vs G3 & G1 vs G3 & G1 vs G3 & G1 vs G3 &
\end{tabular}

Figura 3 - Representação gráfica das médias e respectivos desvios-padrão, dos valores absolutos da DOR À PALPAÇÃO, nos vários momentos do protocolo experimental, nos 3 grupos estudados (G1-Grupo 1; G2-Grupo 2; G3-Grupo 3].

Apesar de G3 ter apresentado uma maior intensidade dos valores médios de dor à palpação, comparativamente a G2, durante todo o estudo, não se verificaram diferenças estatisticamente significativas entre eles. Relativamente às medições da circunferência distal da coxa, como se pode verificar através do quadro 2 , o grupo G1 não sofreu variações estatisticamente significativas ao longo dos vários momentos de avaliação. Nos Grupos G2 e G3 verificaram-se comportamentos semelhantes da circunferência distal da coxa. Em ambos, a circunferência aumentou com a realização dos respectivos programas de exercícios, atingindo valores máximos entre as 24 e 48 horas e apresentaram uma tendência para a normalização a partir das 72 horas.

Na figura 4 encontram-se representados os valores médios da percentagem de variação da circunferência distal da coxa, assim como a análise estatística intergrupal nos vários momentos de avaliação.

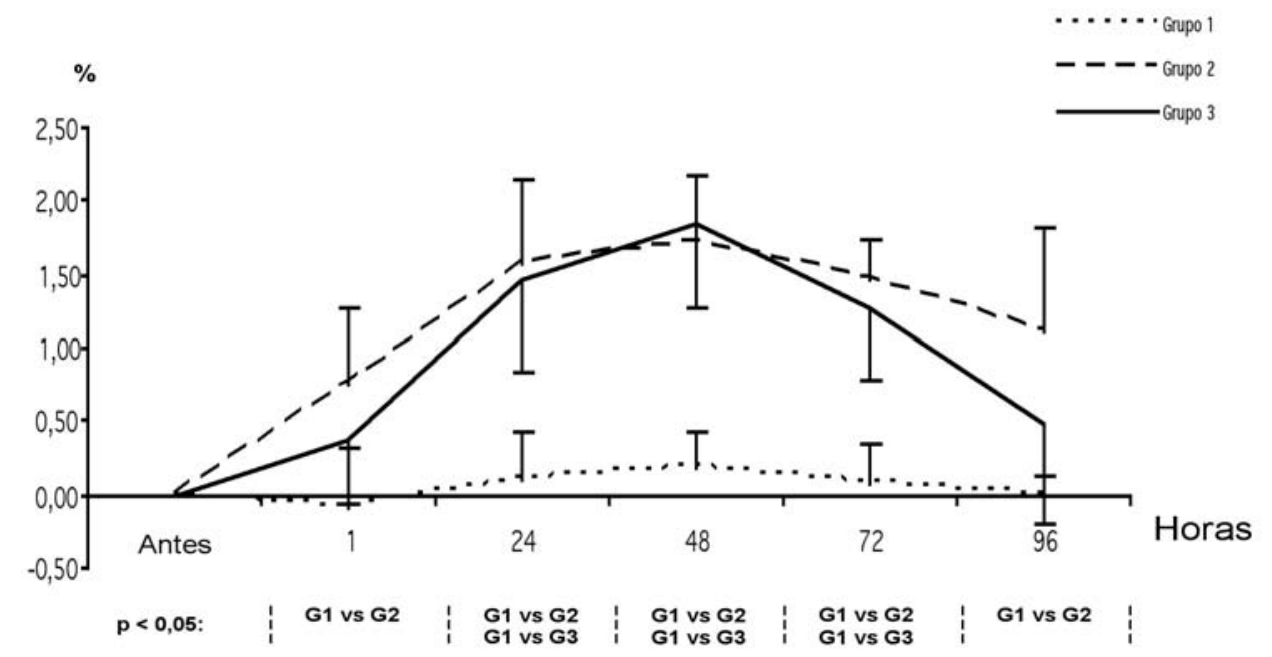

Figura 4 - Representação gráfica das médias e respectivos desvios-padrão da percentagem de variação da CIRCUNFERÊNCIA DISTAL da coxa, nos vários momentos do protocolo experimental, nos 3 grupos estudados (G1-Grupo 1; G2-Grupo 2; G3-Grupo 3). 
Como se analisa, G2 e G3 apresentaram comportamentos semelhantes, sem se terem manifestado diferenças estatisticamente significativas entre eles.

No entanto, comparativamente a G1, enquanto G3 apresentou diferenças significativas entre as 24 e 72 horas $(\mathrm{p}<0.05), \quad \mathrm{G} 2$ manifestou apenas diferenças entre a $1^{\mathrm{a}}$ e as $96^{\mathrm{a}}$ horas $(\mathrm{p}<0.05)$.

Tal como na medição ao nível distal, não houve variações significativas na circunferência proximal da coxa em G1 (quadro 2).

O comportamento ao nível da circunferência proximal da coxa em G2 e G3 foi semelhante. Ambos apresentaram subidas com a realização dos exercícios e manifestaram o maior "pico" de aumento da circunferência às 48 horas. Pode verificar -se, ainda, que G2 e G3 apresentaram valores absolutos significativamente mais elevados, comparativamente à avaliação "antes", a partir das 24 horas $(\mathrm{p}<0.05)$. Estes valores mantiveram-se estatisticamente significativos até final do estudo, para ambos os grupos $(\mathrm{p}<0.05)$, com excepção da avaliação efectuada às 96 horas, momento em que deixou de ser significativo para G3.

A figura 5 representa os valores médios da percentagem de variação da circunferência proximal da coxa, assim como a análise estatística intergrupal nos vários momentos de avaliação.

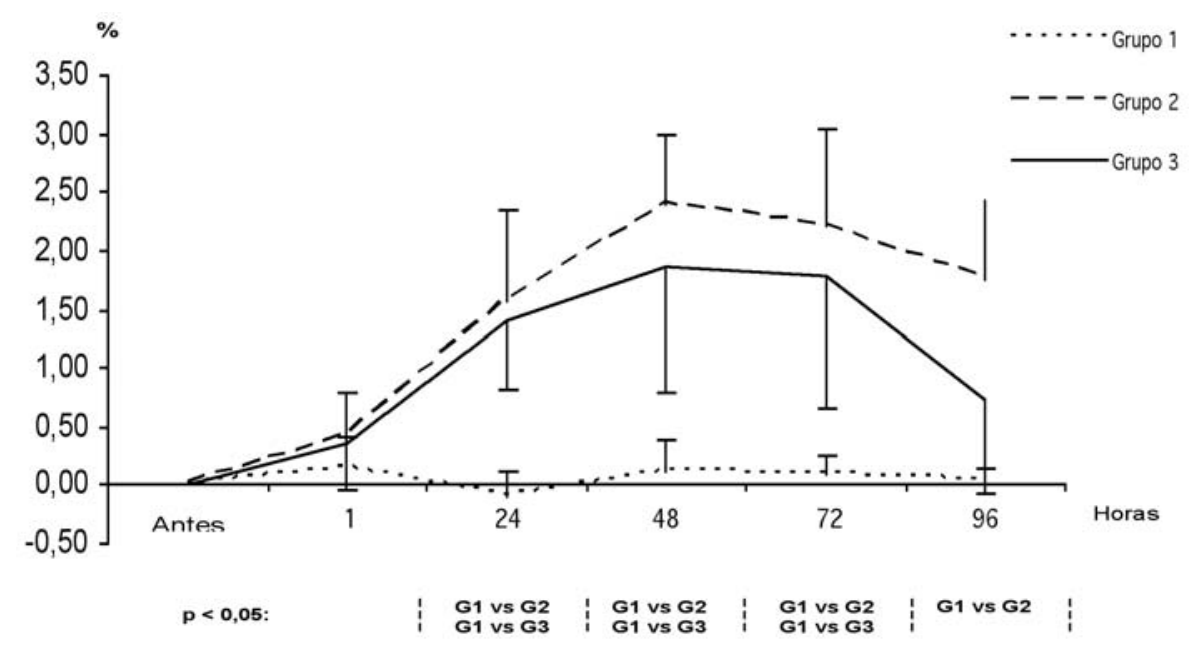

Figura 5 - Representação gráfica das médias e respectivos desvios-padrão da percentagem de variação da CIRCUNFERÊNCIA PROXIMAL da coxa, nos vários momentos do protocolo experimental, nos 3 grupos estudados (G1-Grupo 1; G2-Grupo 2; G3-Grupo 3).

Embora G2 tenha apresentado uma percentagem de variação da circunferência proximal superior, comparativamente a G3, ao longo dos vários momentos de avaliação, essas diferenças não se expressaram significativas do ponto de vista estatístico.

Quanto aos níveis de força, o Grupo G1 não manifestou variações estatisticamente significativas ao longo dos vários momentos de avaliação.

Os grupos G2 e G3 apresentaram comportamentos da força similares, tendo -se registado perdas estatisticamente significativas na $1^{\text {a }}$ hora após o exercício e uma recuperação progressiva nas medições seguintes $(\mathrm{p}<0.05)$. Contudo, a recuperação dos níveis de força, para valores não significativos, foi registada para G3 às 48 horas, e para G2 às 72 horas.

Os valores médios da percentagem de variação do peak torque excêntrico, assim como a análise estatística intergrupal nos vários momentos de avaliação do protocolo experimental, encontram-se representados na figura 6. 


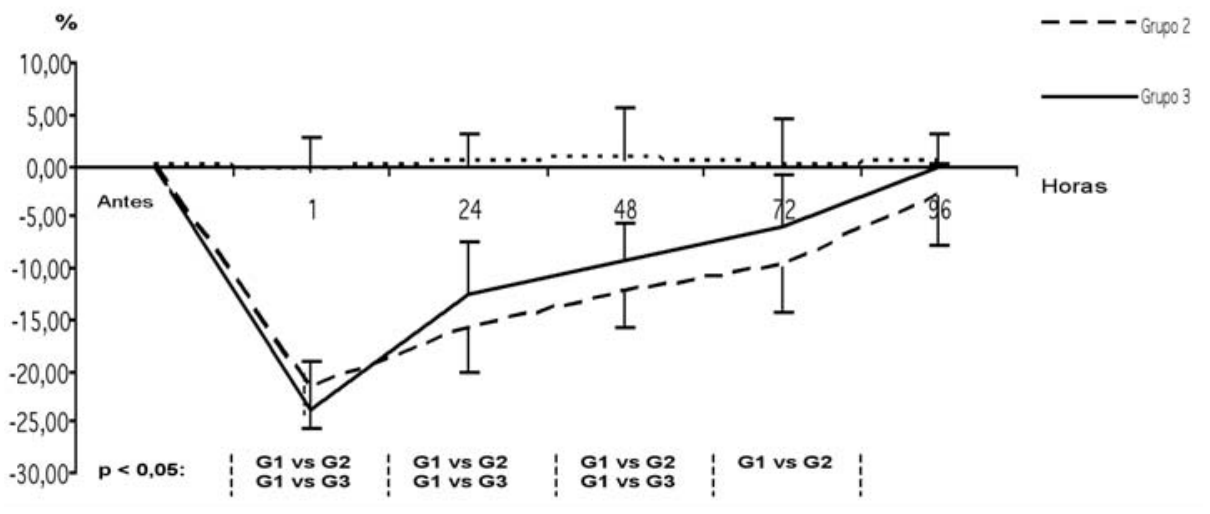

Figura 6 - Representação gráfica das médias e respectivos desvios-padrão da percentagem de variação da FORÇA (N.m), nos vários momentos do protocolo experimental, nos 3 grupos estudados (G1-Grupo 1; G2-Grupo 2; G3-Grupo 3].

Os grupos G2 e G3 apresentaram comportamentos da força idênticos ao longo do estudo, não se tendo verificado diferenças significativas entre eles.

Comparando a percentagem de variação da força de G3 e G2 com G1, verificou-se que G3 manteve défices estatisticamente significativos entre a 1 e as 48 horas $(\mathrm{p}<0.05)$, enquanto que em G2, os défices foram registados entre a 1 e as 72 horas $(\mathrm{p}<0.05)$.

\section{Avaliação bioquímica}

Como se pode obser var através do quadro 2, não se verificam diferenças nos valores da actividade plasmática da CK e da TGO em G1.

Embora G2 e G3 tenham apresentado subidas dos níveis de CK e de TGO ao longo dos vários momentos de avaliação do protocolo experimental, estas não se confirmaram estatisticamente significativas comparativamente à medição "antes".

Os valores médios, assim como a análise estatística intergrupal da actividade plasmática da CK e da TGO, nos vários momentos de avaliação do protocolo experimental, encontram-se representados na figura 7 e 8 .

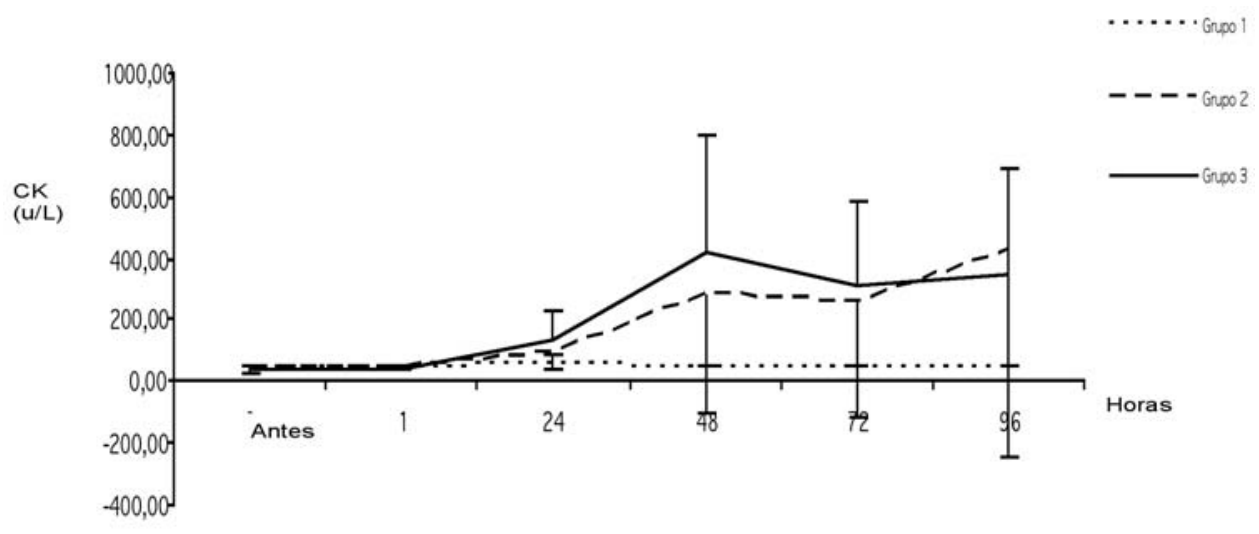

Figura 7 - Representação gráfica das médias e respectivos desvios-padrão, dos valores absolutos de CREATINA KINASE (CK; U/L), nos vários momentos do protocolo experimental, nos 3 grupos estudados. 


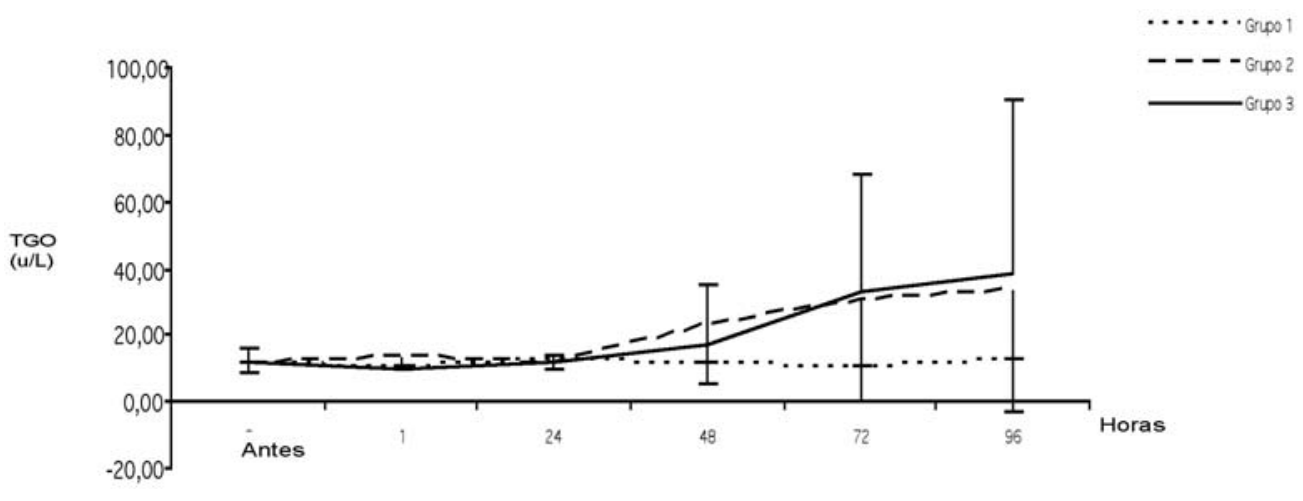

Figura 8 - Representação gráfica das médias e respectivos desvios-padrão, dos valores absolutos de TRANSAMINASE GLUTÂMICA OXALOACÉTICA (TGO; U/I), nos vários momentos do protocolo experimental, nos 3 grupos estudados.

Como se pode analisar, G2 e G3 apresentaram comportamentos similares da actividade plasmática da CK e da TGO, sem diferenças significativas entre eles. Apesar de G1 apresentar um comportamento diferente de $\mathrm{G} 2$ e G3, ao nível da actividade plasmática da CK e da TGO, não se manifestaram significativas do ponto de vista estatístico.

\section{DISCUSSÃO DOS RESULTADOS}

Os resultados encontrados neste estudo não suportam as hipóteses por nós levantadas no início deste trabalho.

De facto, nos resultados obtidos no grupo submetido unicamente ao programa de estiramentos (G1), não foram detectadas variações nos marcadores indirectos que indiciassem qualquer tipo de agressão muscular. O grupo G1 não apresentou qualquer manifestação de dor induzida por palpação ao longo dos vários momentos de avaliação do protocolo experimental. Os resultados registados neste grupo são, assim, contraditórios aos obtidos por Smith et al. (47). Estes autores detectaram manifestações de dor significativas, com os valores a atingirem $3,2 \mathrm{~cm}$ na EVA, 24 horas após a finalização da aplicação dos estiramentos. Verificaram ainda, subidas ligeiras (126,7 U/L), mas significativas, dos níveis de $\mathrm{CK}$, no mesmo período. Também os níveis plasmáticos de CK e TGO não sofreram em G1 alterações ao longo do tempo, contrariamente ao estudo de Smith et al. (47), que indiciassem uma agressão mecânica responsável por alterações estruturais ao nível da fibra muscular, induzida pelo programa de estiramento.
Embora Smith et al. (47) não tenham utilizado a força como parâmetro de avaliação da função muscular, este tem sido extremamente utilizado como indicador indirecto do estado de integridade muscular (6, $7,9,11,17,18,49$ ), sendo mesmo considerado como um indicador seguro de avaliação da integridade muscular (51). Deste modo, os valores constantes em G1 ao longo de todo o estudo, parecem confirmar, também, que o programa de estiramento não induziu agressão muscular.

Por todas estas razões, a realização de exercícios de estiramento estático, por si só, não parece induzir agressão no músculo íntegro.

O grupo G2, submetido unicamente ao programa de exercício excêntrico, apresentou evidências de agressão muscular, tal como pretendido.

A elevação dos níveis de actividade plasmáticos das enzimas musculares CK e TGO, ao longo das medições estipuladas pelo protocolo experimental, sugerem ter havido lesões estruturais ao nível muscular, próprias da realização de um exercício excêntrico extenuante e/ou inabitual (10). Os níveis destas enzimas, apresentaram subidas na sua actividade plasmática a partir das 24 horas e foram aumentando progressivamente ao longo das restantes avaliações previstas pelo protocolo experimental neste grupo. Comportamento similar das enzimas musculares foi também encontrado por Clarkson et al. (11), Lund et al. (28) e Nosak a e Newton (37). No entanto, dada a variabilidade inter-individual da actividade plasmática da CK e TGO, não é possível quantificar com 
segurança o nível da lesão muscular ocorrida através deste indicador $(33,10)$.

Neste grupo (G2), a manifestação da dor ao longo do tempo foi semelhante à encontrada em vários estudos nesta temática $(11,23,52)$. Segundo Armstrong (2), a dor induzida pelo exercício surge habitualmente pela $8^{a}$ hora após a finalização do mesmo, tem o seu pico máximo entre as 24 e 72 horas e, progressivamente, vai diminuindo até ao $5^{\circ}$ ou $7^{\circ}$ dia. De facto, o comportamento da dor à palpação obser vado em G2 foi de encontro ao descrito pelo referido autor.

A origem do desconforto muscular verificado durante ou imediatamente após a realização de um exercício físico, poderá estar relacionada com fenómenos de natureza metabólica e/ou com alteração do fluxo sanguíneo, ao nível dos capilares anexos aos grupos musculares exercitados, motivados pelo aumento das resistências periféricas $(30,34)$. Este mecanismo poderá implicar um aumento do gradiente de pressão nestes capilares, levando a um incremento da saída de líquido plasmático para o interstício e à sua acumulação neste espaço (44), levando à alteração do perímetro, após a finalização do exercício. Contudo, não foram encontradas variações do perímetro nas medições realizadas ao nível da coxa em G2, uma hora após exercício, fazendo sugerir que a agressão muscular induzida neste grupo não teve uma origem predominantemente metabólica.

Assim, a manifestação do desconforto com natureza retardada muscular, verificada no quadro clínico da agressão muscular induzida pelo exercício, parece ter uma outra origem, sugerindo uma relação com a resposta inflamatória causada pela agressão muscular (20, 32, 46). De facto, a manifestação do edema muscular leva a um aumento da pressão nas zonas intersticiais, ao nível dos músculos exercitados (44), estimulando as fibras amielínicas do tipo III e IV responsáveis pela transmissão dos estímulos dolorosos despoletados por factores químicos, mecânicos e térmicos (34).

As variações da circunferência da coxa obtidas em G2, surgiram 24 horas após a finalização do exercício, tiveram o seu pico máximo às 48 horas e, progressivamente, foram diminuindo, sem que se tenha verificado um total restabelecimento na última avaliação. Não foi, assim, encontrada uma semelhança temporal com os resultados obtidos por Nosak a e Clarkson (36), cujo aumento da circunferência apresentou valores máximos entre o $5^{\circ}$ e $10^{\circ}$ dia. A razão para esta diferente expressão temporal poderá estar, eventualmente, relacionada com o tipo de protocolo experimental, nomeadamente com a menor intensidade do exercício excêntrico aplicado neste grupo. Quanto à diferenciação entre a medição proximal e distal, verificámos que a percentagem de variação atingiu valores superiores ao nível proximal, tal como esperado $(24,46)$. Segundo estes autores, a variação da circunferência da coxa, ao nível do ventre muscular, com uma natureza retardada, poderá indicar uma instalação de edema por acumulação de fluidos no meio intersticial, como resposta do processo infamatório. Não foi verificada uma variação significativa da coxa ao nível proximal, na avaliação realizada 1 hora após a finalização do exercício, que pudesse estar relacionada com o aumento da tonicidade e vascularização muscular $(24,47)$.

No que se refere às variações da circunferência distal, os resultados não nos sugerem um aumento de síntese de tecido conjuntivo, como proposto por Smith (46), dado o seu tempo de aparecimento e rápido restabelecimento, relativamente aos valores basais. Acreditamos que a variação a este nível esteja relacionada com um mecanismo idêntico ao da circunferência proximal, pela sua semelhança no comportamento ao longo do tempo.

Relativamente à capacidade de produção de força em $\mathrm{G} 2$, verificou-se uma perda nos valores de peak torque excêntrico 1 hora após a finalização do exercício, o que sugere uma perda da integridade do músculo com a aplicação do respectivo programa de exercícios (51). A percentagem de variação (aproximadamente 22\%) sofrida no peak torque 1 hora após a finalização do exercício, por $\mathrm{G} 2$, e a rápida recuperação, sugere a existência de uma agressão moderada. Para Saxton et al. (42), a lesão muscular poderá implicar uma perda até $50 \%$ dos valores de força e levar 1 a 2 semanas até à sua total recuperação.

Assim, consideramos que o programa cumpriu a agressão muscular pretendida, ou seja, não produziu uma lesão muscular demasiado severa, ao ponto de colocar em causa a aplicação do programa de estiramento por intolerância dos sujeitos e/ou impossibilitar a medição de eventuais alterações clínicas ou 
bioquímicas, ocorridas pela aplicação de uma agressão mecânica de menor intensidade, como a provocada pelo programa de estiramentos.

Uma análise dos resultados entre os grupos G2 e G3, leva-nos a rejeitar a hipótese de que o estiramento estático agrava a manifestação dos quadros clínico e bioquímicos, quando realizado após a agressão muscular induzida pelo exercício excêntrico extenuante e/ou inabitual; no entanto, apesar dos estiramentos não se terem mostrados maléficos, a sua realização após o exercício também não se mostrou vantajosa para a recuperação muscular, ou alívio da sintomatologia.

A análise intergrupal da actividade plasmática das enzimas musculares permite apenas supor que ambos os grupos sofreram agressões semelhantes pelos programas aplicados. A grande variabilidade inter-sujeito, demonstrada pelos elevados desviospadrão, está de acordo com a investigação de outros autores $(9,11,16,43)$. Tal facto, não permite que o aumento dos níveis de actividade plasmática das enzimas musculares seja um indicador indirecto quantitativo da agressão causada.

Tal como os resultados obtidos por Buroker e Schwane (7), High et al. (22) e Lund et al. (28), não se detectaram em G3 diferenças significativas nos níveis de dor, quando aplicado um programa de estiramentos após a agressão pelo exercício, comparativamente a G2.

Deste modo, parece ser de excluir a possibilidade do estiramento ter uma acção ao nível do limiar de excitabilidade das terminações nervosas das fibras amielínicas do tipo III e IV, como sugerido por Armstrong (2). Numa comparação dos níveis de força entre os grupos G2 e G3, verifica-se que os resultados vão de encontro aos obtidos por vários autores $(7,23,40)$, ou seja, não se verificaram diferenças na aplicação de estiramento após o exercício, e são contrários aos referidos por Lund et al. (28), cujos níveis de força diminuíram com a aplicação do estiramento.

Numa comparação entre os grupos G2 e G3 da variação das circunferências ao nível da coxa, é possível verificar uma ausência de diferenças em ambos os níveis de medição, ao longo dos vários momentos de avaliação. Desta forma, não parece ser vantajosa a realização de estiramentos após o exercício, como sugerido por Bobbert et al. (5). Segundo este autor, a realização de estiramentos após o exercício ajudaria na dispersão dos fluidos intersticiais, diminuindo a pressão intramuscular, aliviando a manifestação da sintomatologia dolorosa.

Os resultados encontrados neste estudo deverão, por isso, ter em consideração as características da amostra, bem como o tipo de protocolo experimental, sendo aconselhada alguma prudência nas extrapolações para situações diferentes das verificadas neste trabalho.

\section{CONCLUSÃO}

Face aos resultados obser vados neste estudo, e tendo em consideração os seus objectivos, poderemos concluir que o programa de estiramentos estáticos aplicado neste trabalho, por si só, não parece ter induzido uma agressão muscular esquelética no quadriceps íntegro e, quando realizado neste músculo após a agressão pelo exercício excêntrico, não parece ter agravado as manifestações clínicas e bioquímicas da MEx. Pode verificar-se, ainda, que não parece ter sido vantajosa a aplicação de estiramentos imediatamente após a indução da MEx.

Contudo, a implementação de um programa com sucessivos momentos de aplicação do estiramento estático poderá ter implicações diferentes na agressão muscular induzida pelo exercício. Por isso, sugere-se, em futuras investigações, a realização de estudos que englobem protocolos experimentais com vários momentos de aplicação de estiramentos ao longo do tempo.

\section{CORRESPONDÊNCIA}

\section{Rui Manuel Torres}

Departamento de Fisioterapia

Escola Superior de Saúde de Vale do Sousa

Instituto Politécnico da Saúde Norte

Rua Central de Gandra, 1317

4585-116 Gandra PRD

Portugal

rui.torres@ipsn.cespu.pt 


\section{BIBLIOGRAFIA}

1. Anderson, B.; Burke, E. R. (1991). Scientific, medical, and practical aspects of stretching. Clin. Sports Med. 10: 63-86.

2. Armstrong, R. B.(1984). Mechanisms of exercise-induce delayed onset muscular soreness: a brief review. Med. Sci. Sports Exerc. 16: 529-538.

3. Armstrong, R. B.(1990). Initial events in exercise-induce muscular injury. Med. Sci. Sports Exerc. 22: 429-435.

4. Armstrong, R. B.; Warren, G. L.; Warren, G. A.(1991). Mechanisms of exercise-induced fibre injury. Sports Med. 12: 184-207.

5. Bobbert, M. F.; Hollander, P.; Huijing P. A. (1986). Factors in delayed onset muscular soreness of men. Med. Sci. Sports Exerc. 18: 75-81.

6. Brown, S. J.; Child, R. B.; Day, S. H.; Donnelly, A. E. (1997). Exercise-induce skeletal muscle damage and adaptation following repeated bouts of eccentric muscle contractions. J. Sports Sci. 15: 215-222.

7. Buroker, K. C.; Schwane, J. A. (1989). Does post exercises static stretching alleviates delayed muscle soreness? Phys. Sports Med. 17(6): 65-83.

8. Chan, S. P.; Hong, Y.; Robinson, P. D. (2001). Flexibility and passive resistance of the hamstrings of young adults using two different static stretching protocols. Scand.J. Med. Sci. Sports 11: 81-86.

9. Clarkson, P. M. (1992). Exercise-induce muscle damage-animal and human models. Med. Sci. Sports Exerc 24: 510-521.

10. Clarkson, P. M.; Hubal, M. J. (2002). Exercise-induced muscle damage in humans. Am. J. Phys. Med. Rehabil. 81(Suppl): S52-S69.

11. Clarkson, P. M.; Nosak a, K.; Braun, B. (1992). Muscle function after exercise-induce muscle damage and rapid adaptation. Med. Sci. Sports Exerc. 24: 512-520.

12. Danneck er, E. A.; Koltyn, K. F.; Riley, J. L.; Robinson, M. E. (2002). The Influence of endurance exercise on delayed onset muscle soreness. J. Sports Med. Phys. Fitness. 42: 458 465.

13. DeVries, H. A. (1966). Quantitative electromyographic investigation of the spasm theory of muscle pain. Am.J. Phys. Med. 45: 119-134.

14. Duarte, J. A. R.; Soares, J. M. C. (1990). Sensação retardada de desconforto muscular : etiologia, fisiopatologia, tratamento e prevenção. Rev. Port. Med. Desp. 3: 155-161.

15. Duarte, J. A. R.; Soares, J. M. C. (1991). Etiologia da fadiga muscular: alguns factores condicionantes. Rev. Port. Med. Desp. 9: 165-174.

16. Ebbeling, C. B.; Clarkson, P. M. (1990). Muscle adaptation prior to recover y following eccentric exercise. Eur. J. Appl. Physiol. Occup. Physiol. 60: 26-31.

17. Eston, R. G.; Finney, S.; Baker, S.; Baltzopoulos, V. (1996). Muscle tenderness and peak torque after downhill running a prior bout of isokinetic eccentric exercise. J. Sports Sci. 14: 291-299.

18. Evans, R. K.; Knight, k. L.; Draper, D. O.; Parcell, A. (2002). Effects of warm-up before eccentric exercise on indirect markers of muscle damage. Med. Sci. Sports Exerc, 34: 1892-1899.

19. Feland, J. B.; Myrer J. W.; Merrill, R. M. (2001). Acute changes in hamstring flexibility: PNF versus static stretch in senior athletes. Phys. Ther. Sport 2: 186-193.

20. Friden, J.; Lieber, R. L. (1992). Structural and mechanical basis of exercise-induce muscle injury. Med. Sci. Sports Exerc. 24: 521-530.

21. Herbert, R. D.; Gabriel, M. (2002). Effects of stretching before and after exercising on muscle soreness and risk of injury: Systematic review. BMJ 325: 1-5

22. High, D. M.; Howley, E.T.; Franks, B. D. (1989). The effects of static stretching and warm-up on prevention of delayed onset muscle soreness. Res. Q. Exerc. Sports 60: 357-361.

23. Johansson, P. H.; Lindstrom, L.; Sundelin, G.; Lindstrom, B. (1999). The effects of preexercise stretching on muscular soreness, tenderness and force loss heavy eccentric ex ercise. Scand. J. Med. Sci. Sports 9: 219-225.

24. Jones D. A.; Newham, D. J.; Torgan C. (1989). Mechanical influences on long-lasting human muscle fatigue and delayed muscle- onset pain. J. Physiol. 412: 415-427.

25. Laur, D. J.; Anderson, T.; Geddes, G.; Crandall, A.; Pincivero, D. M. (2003). The effects of acute stretching on hamstring muscle fatigue and perceived exertion. J. Sports Sci. 21: 163-170.

26. Lieber, R. L.; Thornell, L.; Friden, J. (1996). Muscle cytoskeletal disruption occurs within the first $15 \mathrm{~min}$ of cyclic eccentric contraction. J. Appl. Physiol. 80: 278-284.

27. Lieber, R. L.; Woodburn, T. M.; Friden, J. (1991). Muscle damage induced by eccentric contractions of $25 \%$ strain. $J$. Appl. Physiol. 70: 2498-2507.

28. Lund, H.; Vestergaard-P oulsen, P.; Kanstrup, I.L.; Sejrsen, P. (1998). The effect of passive stretching on delayed onset muscle soreness and other detrimental effects following eccentric exercise. Scand. J. Med. Sci. Sports 8: 216-221.

29. Lund, H.; Vestergaard-P oulsen, P.; Kanstrup, I.L.; Sejrsen, P. (1998). Isokinetic eccentric exercise as a model to induce and reproduce pathophysiological alterations to delayed onset muscle soreness. Scand. J. Med. Sci. Sports 8: 208215.

30. MacIntyre, D. L.; Reid, W. D.; McKenzie, D. C. (1995). Delayed muscle soreness: the inflammator y response to muscle injury and its clinical implications. Sports Med. 20: 24-40.

31. Magnusson, S. P.; Aagaard, P.; Simonsen, E. B.; BojsenMoller, F. (1998). A Biomechanical evaluation of cyclic and static stretch in human skeletal muscle. Int. J. Sports Med. 19: $310-316$

32. Malm, C. (2001). Exercise-induced muscle damage and inflammation fact or fiction? Acta Physiol. Scand. 171: 233239.

33. Manfredi, T. G.; Fielding, R. A.; O'R eilly, K. P.; Meredith, C. N.; Lee, H.Y.; Evans, W. J. (1991). Plasma creatine kinase activity and exercise-induce muscle damage in older men. Med. Sci. Sports Exerc. 23: 1028-1034.

34. Miles, M. P.; Clarkson, P. M. (1994). Exercise-induce muscle pain, soreness, and cramps. J. Sports Med. Phys. Fitness 34: 202-216.

35. Miles, M. P.; Ives, J. C. ; Vincent, K. R. (1997). Neuromuscular control following maximal eccentric exercise. Eur. J. Appl. Physiol. 76: 368-374.

36. Nosak a, K.; Clarkson, P. M. (1997). Influences of previous concentric exercise on eccentric exercise induce muscle damage. J. Sports Sci. 15: 477-483.

37. Nosak a, K.; Newton, M. (2002). Concentric or eccentric training effect on eccentric exercise-induce muscle damage. Med. Sci. Sports Exerc. 34: 63-69. 
38. Perrin, D. H. (1993). Isokinetic Exercise and Assessment. Champaign, Il.: Human Kinetic Publisher

39. Rodenburg, J. B.; Bar, P. R.; Boer, R. W. (1993). Relations between muscle soreness and biochemical and functional outcomes of eccentric exercise. J. Appl. Physiol. 74: 29762983.

40. Rodenburg, J.B.; Steenbeek, D.; Schiereck, P.; Bar, P. R. (1994). Warm-up, stretching and massage diminish harmful effects of eccentric exercise. Int. J. Sports Med. 15: 414419.

41. Rosier, E. M.; Iadarola, M. J.; Coghill, R. C. (2002). Reproducibility of pain measurement and pain perception . Pain 98: 205-216.

42. Saxton, J. M.; Clarkson, P. M.; James, R.; Miles, M.; Westerfer, S. C.; Donnelly, A. E. (1995). Neuromuscular dysfunction following eccentric exercise. Med. Sci. Sports Exerc. 27: 1185-1193.

43. Schwane, J. A.; Buckley, R. T.; Dipaolo, D. P.; Atkinson, M. A. L.; Shepherd, J. R. (2000). Plasma creatine kinase responses of 18 to $39 \mathrm{yr}$ old African-American men to eccentric exercise. Med. Sci. Sports Exerc. 32: 370-378.

44. Shellock, F. G.; Fukunaga, T.; Mink, J. H.; Edgerton, V. R. (1991). Acute effects of exercise on MR image of skeletal muscle: concentric vs eccentrics actions. Am. J. Radiol. 156: 659-664.

45. Shellock, F. G.; Prentice, W. E. (1985). Warming-up and Stretching for improve physical performance and prevention of sports-related injuries. Sports Med. 2: 267-278.

46. Smith, L. L. (1991). Acute inflammation: the underlying mechanism in delayed onset muscle soreness? Med. Sci. Sports Exerc. 23: 542-551.

47. Smith, L. L.; Brunetz, M. H.; Chenier, T. C.; McCammon, M. R.; Houmard, J. A.; Franklin, M. E.; Israel, R.G. (1993). The effects of static and ballistic stretching on Delayed Onset Muscle Soreness and Creatine Kinase. Res. Q. Exerc. Sport 64: 103-107

48. Soares, J. M. C.; Duarte, J. A. R. (1989). Miopatia do exercício: etiologia, fisiopatologia e factores de prevenção. Rev. Port. Med. Desp. 7: 187-200.

49. Tiidus, P. M.; Shoemak er, J. K. (1995). Effleurage massage, muscle blood flow and long-term post-e xercise strength recover y. Int. J. Sports Med. 16: 478-483.

50. Trinick, J. (1994). Titin and nebulin: protein rulers in muscle? (Review). Trends Biochem. Sci. 19: 405-409.

51. Warren, G. L.; Lowe, D. A.; Armstrong, R. B. (1999). Measurement tools used in the study of eccentric contraction-induce injury. Sports Med. 27: 43-59.

52. Wessel, J.; Wan, A.(1994). Effect of stretching on the intensity of delayed- onset muscle soreness. Clin. J. Sports Med. 4: 83-87. 